\title{
Circadian regulation of a limited set of conserved microRNAs in Drosophila Maocheng Yang ${ }^{\dagger 1,2,4}$, Jung-Eun Lee ${ }^{\dagger 3}$, Richard W Padgett ${ }^{1,2}$ and Isaac Edery*1,3
}

Address: ${ }^{1}$ Department of Molecular Biology and Biochemistry, Rutgers University, Piscataway, New Jersey, USA, ${ }^{2}$ Waksman Institute, Rutgers University, Piscataway, New Jersey, USA, ${ }^{3}$ Center for Advanced Biotechnology, Piscataway, New Jersey, USA and ${ }^{4}$ National Cancer Institute, National Institutes of Health, Bethesda, Maryland, USA

Email: Maocheng Yang - yangm3@mail.nih.gov; Jung-Eun Lee - jungeunl@eden.rutgers.edu; Richard W Padgett - padgett@waksman.rutgers.edu; Isaac Edery* - edery@cabm.rutgers.edu

* Corresponding author †Equal contributors

Published: 19 February 2008

BMC Genomics 2008, 9:83 doi:10.1/86/147|-2/64-9-83
Received: 26 September 2007

Accepted: 19 February 2008

This article is available from: http://www.biomedcentral.com/I47I-2/64/9/83

(C) 2008 Yang et al; licensee BioMed Central Ltd.

This is an Open Access article distributed under the terms of the Creative Commons Attribution License (http://creativecommons.org/licenses/by/2.0), which permits unrestricted use, distribution, and reproduction in any medium, provided the original work is properly cited.

\begin{abstract}
Background: MicroRNAs (miRNAs) are short non-coding RNA molecules that target mRNAs to control gene expression by attenuating the translational efficiency and stability of transcripts. They are found in a wide variety of organisms, from plants to insects and humans. Here, we use Drosophila to investigate the possibility that circadian clocks regulate the expression of miRNAs.
\end{abstract}

Results: We used a microarray platform to survey the daily levels of $D$. melanogaster miRNAs in adult heads of wildtype flies and the arrhythmic clock mutant $\mathrm{cyc}^{\mathrm{O}}$. We find two miRNAs (dmemiR-263a and -263b) that exhibit robust daily changes in abundance in wildtype flies that are abolished in the $\mathrm{cyc}^{\mathrm{Ol}}$ mutant. dme-miR-263a and $-263 \mathrm{~b}$ reach trough levels during the daytime, peak during the night and their levels are constitutively elevated in $c y \mathrm{c}^{0 \mathrm{l}}$ flies. A similar pattern of cycling is also observed in complete darkness, further supporting circadian regulation. In addition, we identified several miRNAs that appear to be constitutively expressed but nevertheless differ in overall daily levels between control and $c y \mathrm{c}^{\mathrm{O}}$ flies.

Conclusion: The circadian clock regulates miRNA expression in Drosophila, although this appears to be highly restricted to a small number of miRNAs. A common mechanism likely underlies daily changes in the levels of dme-miR-263a and -263b. Our results suggest that cycling miRNAs contribute to daily changes in mRNA and/or protein levels in Drosophila. Intriguingly, the mature forms of dme-miR-263a and -263b are very similar in sequence to several miRNAs recently shown to be under circadian regulation in the mouse retina, suggesting conserved functions.

\section{Background}

Circadian rhythms (approx. $24 \mathrm{hr}$ ) are governed by cellular "clocks" or pacemakers that can be synchronized to the daily and seasonal changes in external time cues (zeitgebers), most notably visible light and ambient temperature (reviewed in, [1]). This endogenously driven timing sys- tem enables life forms to anticipate environmental transitions, perform activities at biologically advantageous times during the day and undergo characteristic seasonal responses. An important challenge is to gain a better understanding of the biochemical and cellular bases underlying clocks. 
Despite the wide variety of overt rhythms manifested by different species (e.g., from flowering in plants to wakesleep cycles in humans), there is remarkable similarity in the underlying timing mechanisms. A core feature is based on species or tissue specific sets of 'clock' genes, whose RNA and protein products participate in interconnected positively and negatively acting transcriptional-translational feedback loops $[2,3]$. As a result of the design principles inherent in these autoregulatory molecular loops, one or more of the core clock RNA and protein products manifest 'self-sustained' daily rhythms in abundance that are instrumental for normal clock progression. The central clock transcription factors that participate in their own cyclical expression and that of other core clock genes also either directly or indirectly drive daily oscillations in the expression of many downstream effector genes. Microarray profiling studies indicate that $\sim 1-10 \%$ of the genes expressed in a particular animal cell type undergo circadian fluctuations, demonstrating the strong influence of clocks in global gene expression [4-6]. Indeed, malfunctions in mammalian clock function have been linked to many diseases including cancer, metabolic syndromes and affective disorders [7-9].

Our understanding of clock mechanisms in general and mammalian ones in particular have been strongly based on findings using Drosophila melanogaster as a model system [1]. Key components of the intracellular clock mechanism in this species include PERIOD (Drosophila PER; dPER), TIMELESS (TIM), CLOCK (CLK) and CYCLE (CYC). CLK and CYC are transcription factors of the basichelix-loop-helix (bHLH)/PAS (르-A Arnt- $\underline{\text { Sim) }}$ superfamily that heterodimerize to stimulate the daily transcription of dper and tim, in addition to other clock and downstream genes. After a time delay, dPER and TIM enter the nucleus where the association of dPER with the CLK-CYC heterodimer leads to the inhibition of CLK-dependent transcriptional activity. After several hours the levels of TIM and dPER undergo sharp decreases, relieving autoinhibition and initiating another round of CLK-CYC-dependent transcription. Mutations that either inactivate or severely impair the activities of $d$ per (e.g., per ${ }^{01}$ ), tim (e.g., tim $^{0}$ ) cyc (e.g., $\left.c y c^{01}\right)$, or $C l k$ (e.g., $\left.C l k^{\mathrm{rk}}\right)$, abolish molecular and behavioural rhythms [10].

MicroRNAs (miRNAs) are single-stranded RNA species of $\sim 22$ nucleotides that are derived by processing from a larger precursor and are found in a wide variety of organisms, from plants to insects and humans (recently reviewed in [11]). In animals, miRNAs usually have imperfect complementarity to elements in the 3' untranslated regions (UTRs) of mRNA targets, leading to posttranscriptional regulation of the target mRNA by inhibition of translation and/or stimulation of degradation [1214]. It is thought that miRNAs mainly function to fine- tune the levels of key proteins. At present, estimates suggest there are about 100 miRNA encoding genes in insects, and over 400 in mammals. Although a comprehensive list of mRNAs targeted by miRNAs is still lacking in any organism, computational analysis suggests that a single miRNA could target 100s of genes [15]. Recent studies indicate that miRNAs have diverse physiological roles, such as apoptosis, homeobox regulation, viral infection, and development $[11,16]$. Drosophila has provided a powerful genetic and genomic model system to understand the biological roles of miRNAs $[17,18]$. Herein, we show that several miRNAs in Drosophila are clock-regulated, raising the possibility that they play fundamental roles in circadian systems.

\section{Results \\ A limited number of miRNAs exhibit circadian regulation in Drosophila heads}

To determine whether miRNAs exhibit daily changes in abundance we entrained control D. melanogaster flies $(y w)$ to standard $12 \mathrm{hr}$ light/12 hr dark cycles [12:12LD; where zeitgeber time 0 (ZTO) is defined as beginning of the light phase] at $25^{\circ} \mathrm{C}$ and collected flies at $6 \mathrm{hr}$ intervals during the third LD cycle (i.e., ZT1, 7, 13 and 19). In addition, we analyzed the well-characterized $c y c^{01}$ arrhythmic mutant [19]. The wildtype control and mutant $c y c^{01}$ flies used here are in the same $y w$ genetic background and were treated contemporaneously. RNA was prepared from adult heads, which contain the key pacemaker neurons driving rhythmic behavior [20] and are routinely used to evaluate cycling profiles of mRNAs in Drosophila[5,21]. We used the miRMAX microarray platform with the Array900 miRNA Direct Labeling System [22] that features dimer probes complementary to mature or abridged miRNA sequences of all the identified miRNAs of Drosophila according to the miRBase version 5.1 [23]. For each time point, three independent experiments were analyzed and data pooled (Fig. 1). The Gene Expression Omnibus (GEO) accession number for our microarray raw data is GSE10005.

To identify clock-regulated miRNAs we applied several criteria. First, there had to be at least a statistically significant difference between the lowest and highest signal intensity of the four time points analyzed in wildtype flies. We applied the stringent test of ANOVA with FDR (5\%) to compare within each genotype as a function of time, and between the $y w$ control and $y w ; ; c y c c^{01}$ (herein, more simply refereed to as $c y c^{01}$ ) mutant groups. Second, a bona-fide clock-regulated miRNA should not exhibit statistically significant difference between the four time points in $c y c^{01}$ flies; i.e., levels should remain constant throughout a daily cycle--as is observed for clock-controlled mRNAs in this mutant $[19,24]$. Finally, we required a minimum dif- 

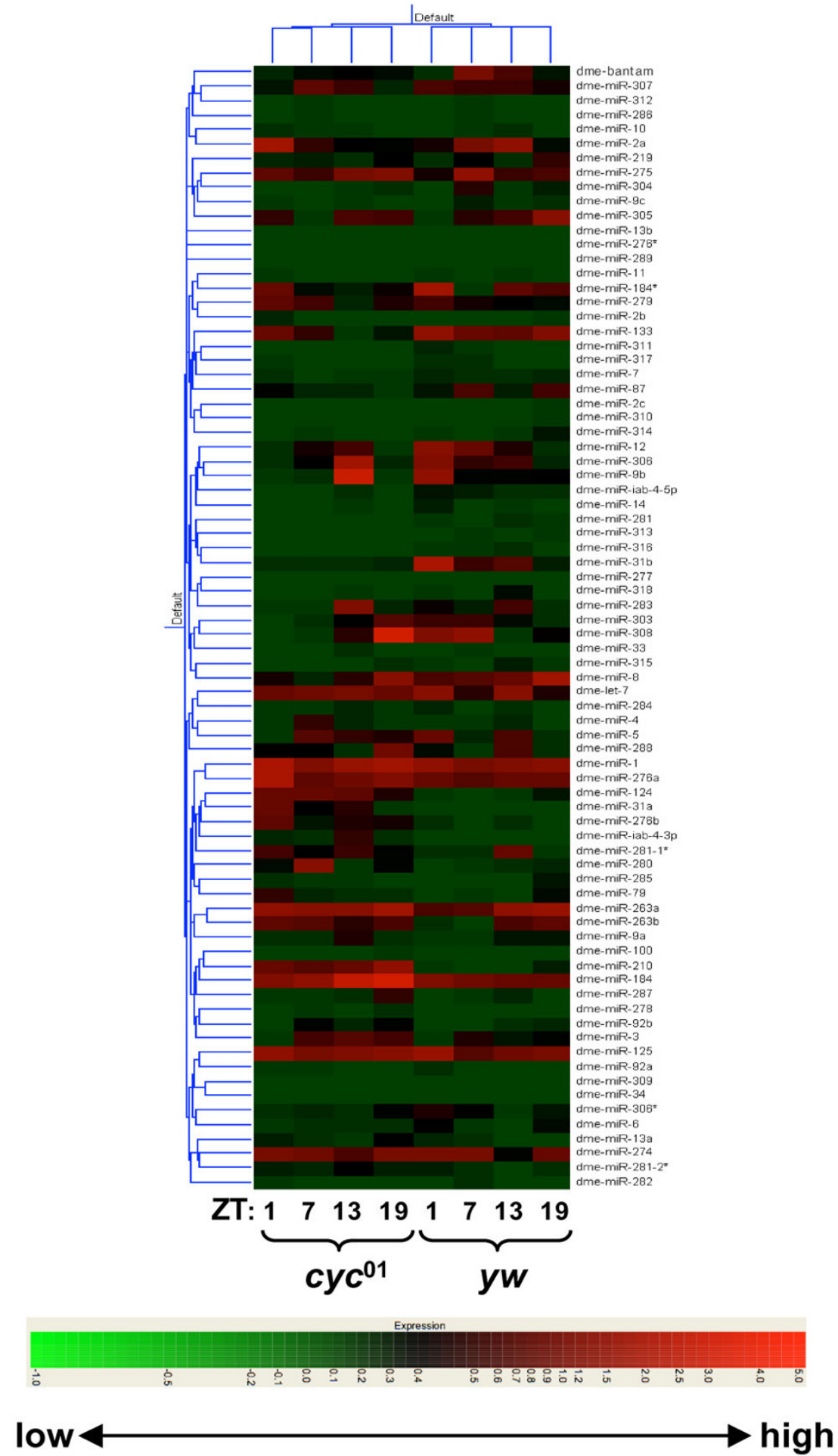

Figure I

Heatmap of Drosophila miRNA expression as a function of daily time and in cyc ${ }^{01}$ flies. Colorgram depicts the relative levels of miRNAs (high, red; average, black; low, green--as indicated by color bar, bottom) and summarizes hierarchical clustering of daily light-dark patterns of miRNAs in control $(y w)$ and mutant $\left(y w ; ; c y c^{0 I}\right)$ flies using Genespring GX (Agilent, Santa Clara, CA). Flies were collected at ZTI, 7, 13 and 19, as indicated (bottom of panel). 
ference of $50 \%$ between peak and trough values in wildtype flies.

We obtained significant signals from 78 Drosophila melanogster miRNAs (Fig. 1). Of these, only two miRNAs (dme-miR-263a and -263b) in wildtype control flies exhibited daily abundance changes that were statistically significant using the ANOVA with 5\% FDR test (Fig. 2A and $2 \mathrm{~B}$ ). Importantly, the levels of miR-263a and -263b were constant throughout a daily cycle in the $c y c^{01}$ mutant, indicative of bona-fide circadian regulation (Fig. 2A and 2B). Intriguingly, both miRNAs attain trough levels during the daytime hours and have constitutively elevated levels in the $c y c^{01}$ mutant, suggesting a common mechanism underlying their clock regulation (see Discussion). We confirmed that both dme-miR-263a and -263b cycle using quantitative RT-PCR (qRT-PCR) and also showed that these oscillations persist in constant dark conditions (Fig. 2C and 2D). Although it is not clear why the daily profiles are slightly different when comparing results obtained using the microarray platform or qRT-PCR, the combined results clearly indicate circadian regulation in the levels of dme-miR-263a and -263b.

No miRNAs that we evaluated showed significant differences in levels as a function of time in $c y c^{01}$ flies, suggesting that (at least in the absence of a functional clock) the light-dark cycle has little to no direct effect on the expression of miRNAs in Drosophila heads. We also identified miRNAs that do not exhibit statistically significant changes throughout a daily cycle in wildtype flies but nonetheless show differences in average daily levels when compared to $c y c^{01}$ flies (Fig. 3). In most cases, the average daily values in $c y c^{01}$ flies were higher compared to control flies. Of these, dme-miR-124 showed a pattern very similar to that of dme-miR-263a and -263b, exhibiting trough levels during the mid-day that were followed by increases during the early to late-night in wildtype flies and constantly elevated levels in the $c y c^{0}$ mutant (Fig. 3B). Although the differences in daily levels for miR-124 in wildtype flies do not reach significance even when less stringent criteria was applied $(\mathrm{p}=0.0813$, ANOVA without FDR), it is possible that miR-124 undergoes low amplitude circadian oscillations in abundance. Likewise, miR-31a (Fig. 3F) might also exhibit low amplitude cycling similar to that of miR-124.

\section{Possible circadian-relevant targets of dme-miR-263a and 263b}

Experimental and computational studies have shown that several hundred different mRNAs can be targeted by each miRNA $[15,25]$. As an initial attempt to identify possible targets for the miRNAs that either show circadian regulation or differences in overall daily levels between control and $c y c^{01}$ flies (Figs. 2 and 3) we used four readily availa- ble web-based prediction programs that contain information on D. melanogaster (i.e., PicTar [26], miRBase [23], TargetScan [27] and EMBL [28]).

Cycling mRNAs that were previously identified based on whole genome expression profiling [29-34] are found among the predicted targets of the clock-regulated miRNAs we detected in this study (data not shown). This is to be expected based simply on the many clock regulated genes reported to date, the possibility that a single miRNA can target many different transcripts and the use of multiple prediction programs. However, although hundreds of circadian regulated mRNAs have been identified in Drosophila using microarray-based strategies, there is little overlap between the different studies, except for the highamplitude core clock transcripts [5,31,35]. In addition, miRNAs apparently function in animals by mainly regulating translational efficiency $[13,14]$, suggesting that constitutively expressed mRNAs are equally good targets of miRNAs to generate daily fluctuations in protein levels. Thus, we limited our focus of possible targets to genes that function within the central clock mechanism or with characterized roles in the Drosophila circadian timing system (Table 1).

Using this more limited search, we noted that $\mathrm{Clk}$ might be a target of both miR-263a and -263b (Table 1). However, this was only predicted by TargetScan and might be an artefact of using a Clk sequence with a premature translation stop codon (i.e., the region in question lies upstream to the normal translation stop codon). In addition, although Clk RNA levels cycle [36], the total abundance of the protein is constant throughout a daily cycle $[37,38]$. Perhaps a more promising candidate is clockwork orange ( $c w o$ ), which is a transcriptional repressor recently shown to modulate circadian rhythms in Drosophila [3941]. Two independent search programs (Pictar and EMBL) predict that cwo might be targeted by miR-263b. Expression of $c w o$ is directly driven by CLK-CYC and exhibits daily RNA cycles that peak in the early night with trough values attained during the late night/early day [39-41]. miR-263b has a similar RNA profile as cwo, raising the possibility that it functions to attenuate translation of $\mathrm{cwo}$ transcripts as they accumulate during the night.

Other possible targets of miR-263a and miR-263b include doubletime (dbt) and twins (tws), respectively. DBT is the Drosophila homolog of casein kinase $1 \varepsilon(\mathrm{CK} 1 \varepsilon)$, whereas TWS is a protein phosphatase type $2 \mathrm{~A}$ regulator. Both play important but presumably opposing or balancing roles in the Drosophila circadian system by targeting key clock proteins, such as dPER and CLK (reviewed in, [42]). Prior work has shown that at least one isoform of the tws transcripts cycles in abundance, rising during the late day and decreasing during the night, while remaining constitu- 

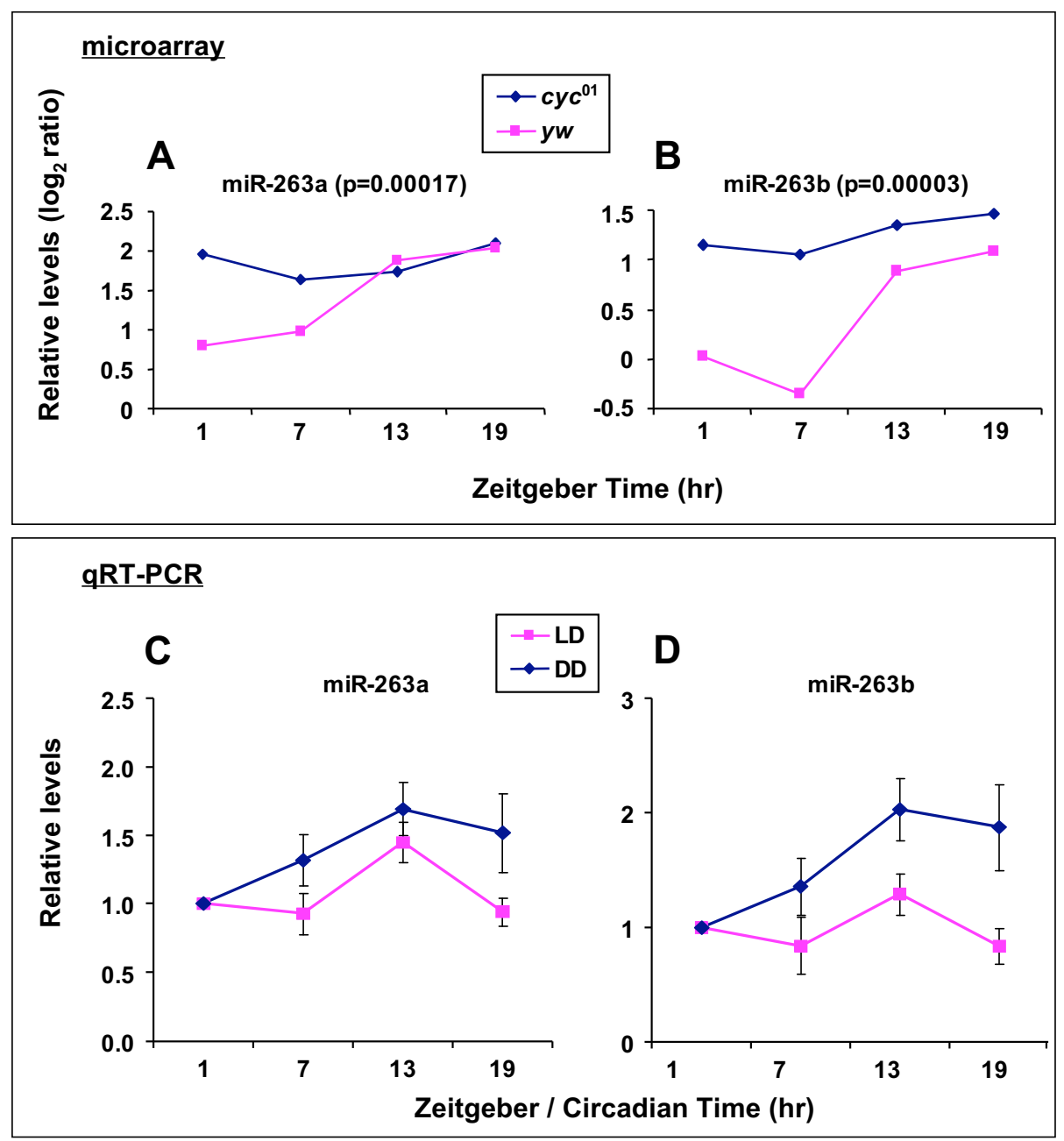

\section{Figure 2}

MiRNAs showing significant differences in levels as a function of daily time within the $y$ w control group. (A, B) $Y w$ or $y w ; ; c y c^{0 I}$ flies were collected at the indicated times during the third LD cycle. LMW RNA was prepared from head extracts and subjected to miRNA microarray profiling. Shown are the miRNAs with statistically different levels as a function of time within the $y$ w group but not within the $y$ w;; $c y c^{01}$ group. P values are indicated for each miRNA. (C, D) Canton-S flies were collected at the indicated times during either the third LD or the first day of DD. Total RNA was prepared from head extracts and subjected to quantitative RT-PCR (qRT-PCR). Results reflect the average of four replicates from two independent experiments. Error bars are S.E.M.

tively low in the $c y c^{01}$ mutant [43]. Intriguingly, miR-263b expression is essentially anti-phase to that of the cycling tws transcript and is constantly high in $c y c^{01}$ flies (Fig. 2). Thus, miR-263b could amplify daily oscillations in the levels or translational efficiency of tws RNA. While speculation is tempting, just one out of the four prediction programs identified $d b t$ and tws. Finally, the physiological significance of targeting $d b t$ expression in a circadian manner by miRNAs is suspect because neither $d b t$ mRNA nor protein levels cycle [44].
With regards to the other class of miRNAs that show differences in overall daily levels between wildtype and $c y c^{01}$ flies (Fig. 3), we noted several clock-relevant genes as putative targets (Table 1). A possibly noteworthy finding is that casein kinase 2 (CKII), which has a role in the Drosophila clock $[45,46]$, is predicted to be a target of miR133, miR-210 and miR-276b. It is thought that the presence of multiple miRNA interaction sites allows for exquisite control of target mRNA expression [47]. Nonetheless, only a single prediction program (in this case, Sanger) identified CkII as a potential target of these miRNAs. Besides cwo, the only clock gene that was predicted by 

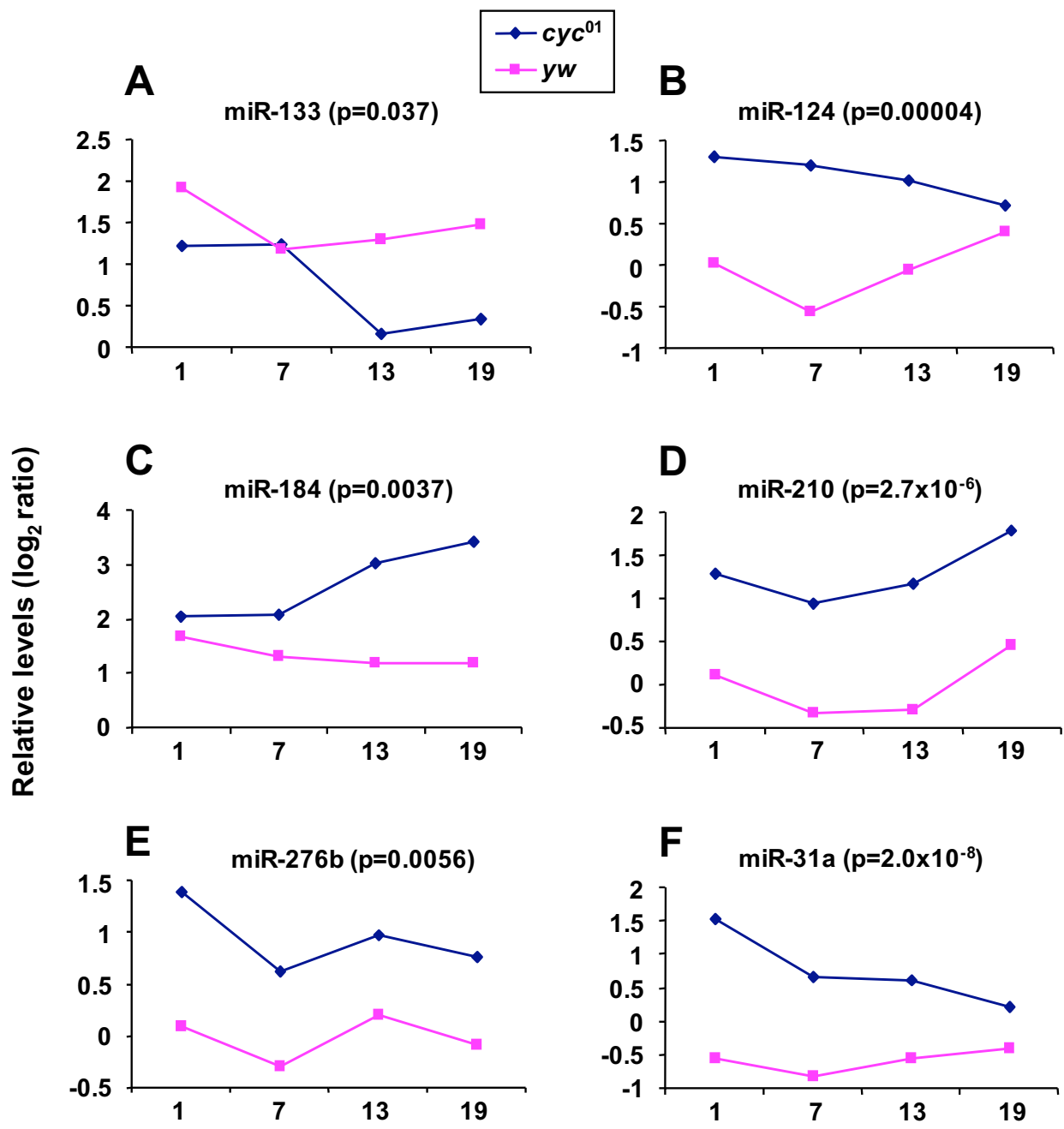

\section{Zeitgeber Time (hr)}

\section{Figure 3}

MiRNAs showing significant differences in overall daily levels between $y w$ and $c y c^{0 I}$ flies. $Y w$ or $y$ w;;cycll flies were collected at the indicated times during the third LD cycle. LMW RNA was prepared from head extracts and subjected to miRNA microarray profiling. Shown are the miRNAs with statistically different overall daily levels between the $y w$ and $y$ $w ; ; c y c^{01}$ groups. $P$ values are indicated for each miRNA.

more than one program as a potential target of a single miRNA was tim, a candidate target of miR-276b (Table 1). Clearly, future studies will be required to verify if any of the miRNAs identified in this study play roles in the clock and/or rhythmic expression.

\section{Discussion}

Our results indicate that the levels of a limited number of miRNAs in D. melanogaster exhibit robust circadian regulation. Of the 78 miRNAs that we probed by expression profiling only dme-miR263a and -263b displayed strong evidence of circadian regulation (with possible weak cycling for dme-miR-124). Importantly, the daily cycles in dme-miR263a and $-263 \mathrm{~b}$ were abolished in the $c y c^{01}$ mutant, and persisted in constant dark conditions (Fig. 2). These results indicate that changes in the amounts of miR263a and -263b are not merely driven by daily light-dark cycle but are dependent on a functional clock. Moreover, we did not detect other miRNAs that display robust cycling profiles in daily light-dark cycles, suggesting that the expression of miRNAs in D. melanogaster is largely insensitive to photic signals. However, we cannot rule out transient effects of light. In addition, it is possible that highly stable miRNAs, despite circadian regulation in 
Table I: Predicted clock-relevant targets for miRNAs identified in this study

\begin{tabular}{|c|c|}
\hline miRNA & Target $^{\mathbf{a}}$ \\
\hline dme-miR-263a & Clk $[\mathrm{T}]^{\mathrm{b}}, \mathrm{dbt}[\mathrm{P}]$, tws $[\mathrm{T}]$, slo $[\mathrm{S}]$ \\
\hline dme-miR-263b & Clk $[\mathrm{T}], \mathrm{Cwo}[\mathrm{P}, \mathrm{E}]$ \\
\hline dme-miR-3 la & - \\
\hline dme-miR-I 24 & jet $[\mathrm{S}]$ \\
\hline dme-miR-I33 & $C_{k l l} \alpha[\mathrm{S}]$, slo $[\mathrm{S}]$ \\
\hline dme-miR-184 & - \\
\hline dme-miR-2I0 & $\operatorname{per}[\mathrm{S}], C_{k} \| l \beta[\mathrm{S}]$ \\
\hline dme-miR-276b & $\operatorname{tim}[\mathrm{S}, \mathrm{T}], C_{k} \| l \beta[\mathrm{S}]$ \\
\hline
\end{tabular}

aThe predicted targets were limited to the following clock relevant genes (annotation symbol); dper (CG2647), tim (CG3234), Clk (CG739I), cyc (CG8727), vri (CGI4029), Pdpl (CGI7888), cwo (CGI7I00), dbt (CG2048), Ckll $\alpha$ (CGI7520), Ckll $\beta$ (CGI5224), sgg (CG262I), slmb (CG34I2), tws (CG6235), wdb (CG5643), mts (CG7109), jet (CG8873), cry (CG3772), slo (CGI0693) and pdf (CG6496)

bThe following abbreviations are used: P, Pictar; S, miRBase (Sanger);

T, TargetScan; E, EMBL.

expression, would not exhibit daily cycles in abundance. Also, as an initial foray into determining whether miRNA expression in Drosophila is under circadian regulation we used total head extracts as a source for miRNA isolation, which could mask cell or tissue specific temporal regulation. Despite these caveats the limited number of cycling miRNAs strongly suggests that these molecular rhythms mainly arise at the level of transcriptional regulation and not global oscillations in the miRNA-processing machinery.

At least two interconnected transcriptional feedback loops operate within the core clock mechanism in Drosophila [48]. In the 'major' loop, CLK and CYC form a heterodimer that binds E-box containing elements found in 5 ' regulatory regions of $d$ per, tim and vrille (vri), in addition to numerous other downstream effector genes. While dPER and TIM cooperate to negatively regulate CLK-CYC transactivation, VRI functions in an interacting transcriptional loop by binding to 5' upstream regulatory elements on $C l k$, an event that inhibits Clk expression. As a result of the molecular logic underlying the intertwined transcriptional circuitry, dper, tim and vri mRNA levels follow a similar temporal profile peaking in the early night, whereas Clk transcripts exhibit essentially anti-phase cycling [36].

MiR-263a and -263b have similar daily profiles, with peak levels in the early to mid-night (between ZT/CT13 and ZT/ CT19) and trough amounts reached during the early to mid-day (ZT/CT1-7) (Fig. 2). Moreover, both miRNAs are constitutively expressed at peak levels in the $c y c^{01}$ mutant, suggesting a common mechanism is controlling daily changes in the levels of miRs $-263 \mathrm{a}$ and $-263 \mathrm{~b}$. The daily patterns of changes in the levels of miR-263a and $-263 \mathrm{~b}$ are somewhat reminiscent of mRNA targets directly regulated by CLK-CYC. However, whereas the levels of bonafide direct targets of CLK-CYC, such as dper and tim, are reduced in $c y c^{01}$ flies $[19,24]$, the abundance of miR-263a and $-263 \mathrm{~b}$ are pegged at peak amounts (Fig. 2). These considerations raise the possibility that the circadian expression of miR-263a and -263b is not directly driven by core clock transcription factors. For example, CLK-CYC might stimulate the rhythmic expression of a transcriptional inhibitor that blocks expression of miR-263a and $-263 b$ during the day.

Besides targeting individual genes, clusters of genes can be co-ordinately regulated by the circadian clock in Drosophila, presumably as a result of temporal changes in chromatin remodelling [33]. In a somewhat analogous manner, recent evidence shows that multiple unique miRNAs can be generated and co-regulated from a single primary miRNA (pri-miRNA) transcript $[49,50]$. However, even though miR-263a and $-263 \mathrm{~b}$ are paralogous genes in the same family (miR-263) with very similar mature sequences (Fig. 4A), they are found on the second and third chromosomes, respectively. The fact that these two miRNAs have closely related target sites and undergo similar circadian regulation suggests that they have parallel functions in contributing to rhythms in the expression of an overlapping set of genes. Nonetheless, recent work comparing 12 Drosophila genomes suggest that some of the miRNA sequences currently in use need to be revised, which could result in marked changes in predicted targets [51]. This could be especially relevant to this study as evolutionary evidence suggests a different $5^{\prime}$ end for miR263a [51].

Although we also identified several (apparently) noncycling miRNAs with statistically different overall daily levels between wildtype and $c \gamma c^{01}$ mutants (Fig. 3), the physiological significance of these results is presently not clear. Nonetheless, whole genome microarray profiling in Drosophila has shown that mutations in core clock transcription factors not only abolishes rhythmic expression but can lead to changes in the basal levels of many constitutively expressed mRNAs [34]. Future work will be instrumental in identifying in vivo targets of the miRNAs identified in this study and evaluate their putative roles in the Drosophila circadian system.

While this manuscript was in preparation, evidence of miRNA cycling and roles in the clock were shown in mammals. One study identified miR-132 and miR-219 as being clock regulated in the suprachiasmatic nucleus (SCN), the 'master clock' in mammals [52]. In addition, they also showed that miR-132 plays a role in circadian photic entrainment, whereas miR-219 modulates period length. 
A

dme-miR-263a GUUAAUGGCACUGGAAGAAUUCAC || || ||||| | ||||||||| dme-miR-263b CUUGGCACUGGGAGAAUUCAC

B

mouse miR-182 (3)

(3) UGGCAAUGGUAGAACUCAC (21)

| | | | || | || | || |

dme-miR-263a GUUAAUGGCACUGGAAGAAUUCAC

| | | | | |||| | | || || || |

mouse miR-183 (2) AUGGCACUGGUAGAAUUCAC (21)

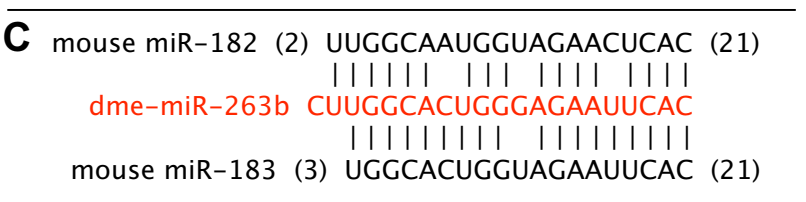

D dme-miR-124 UAAGGCACGCGGUGAAUGCCAAG | | | | | | | | | | | | | | | | | | | |

mouse miR-124 (1) UAAGGCACGCGGUGAAUGCC (20)

\section{Figure 4}

Similarity between some of the miRNAs identified in this study and recently described miRNAs that cycle in mammals. Shown are the alignments between miRNAs identified in this study and possible mammalian orthologs that were recently described by Xu et al. (2007) as cycling in the mouse retina. The entire sequences of the mature forms of Drosophila miRNA are shown in different colors, whereas mouse sequences are shown in black. The identity of the miRNA is shown at left; number in brackets signifies the nucleotide position of the mature mouse miRNA starting from left ( $\left.5^{\prime}\right)$ to right ( $\left.3^{\prime}\right)$. Sequences used were those in the Sanger miRBase as of December 2007.

In another study, several miRNAs specifically expressed in the mouse retina exhibited daily oscillations in levels [50]. Among the subgroup of miRNAs showing circadian regulation in the retina were members of the miR-183/96/ 182 cluster. We used the miRBase database to determine if any of the miRNAs we identified are similar to the recently described cycling miRNAs in mammals. We used the mature sequences of the D. melanogaster miRNAs as queries to search the database for vertebrate miRNAs with similar sequences. Although potentially coincidental, the mature sequence for miR-183 in vertebrates is very similar to that of both $D$. melanogaster dme-miR-263a and miR263b (Figs. 4B and 4C). The D. melanogaster dme-miR$263 \mathrm{~b}$ is also similar to the vertebrate miR-182 (three mismatches over the length of 20 aligned nucleotides). Finally, the D. melanogaster dme-miR-124 is similar to vertebrate miR-124a (Fig. 4D), which also cycled in the mouse retina [50]. Because we probed whole heads where the majority of the clocks reside in the compound eyes [53], this might have contributed to the surprising overlap in cycling miRNAs between our study and those identified in the mouse retina. Of the miRNAs we identified only miR-276b appears not to be conserved in vertebrates.
Thus, our results raise the interesting possibility that similar miRNAs have conserved functions in the circadian systems of vertebrates and insects, perhaps in a tissue-specific manner.

\section{Conclusion}

A small proportion of miRNAs in Drosophila exhibit robust clock-regulated rhythms in abundance. Our results suggest that for some proteins, daily changes in their levels are modulated by miRNA-mediated post-transcriptional regulation. It is likely that the action of miRNAs in rhythmic expression is to provide a fine-tuning mechanism that coordinates with transcriptional and post-translational pathways to generate an oscillatory system that can adjust the levels of specific proteins to values that are appropriate for a given time of day.

\section{Methods}

\section{Fly strains and treatment}

Drosophila strains used were descendants of Canton-S, yw and $\gamma w ; ; c y c c^{01}$ strains previously described [19]. Flies were maintained in standard media at $25^{\circ} \mathrm{C}$. For collections, approximately 30-100 young (2-5 day old) flies were placed in vials that were incubated at $25^{\circ} \mathrm{C}$ for three to four days in standard $12 \mathrm{hr}$ light-12 hr dark cycles [LD; where zeitgeber time 0 (ZT0) is defined as lights-on], and in some cases followed by complete darkness [DD; where circadian time 0 (CT0) is defined as subjective lights-on]. Flies were collected by freezing in dry ice during the third or fourth LD cycle or the first DD cycle at the following times; ZT/CT1, 7, 13 and 19. Subsequently, heads were isolated from the frozen flies and kept at $-70^{\circ} \mathrm{C}$ until further processed, as described below.

\section{RNA preparation and labelling}

Adult fly heads were flash frozen and ground into fine powder in liquid nitrogen for miRNA microarray analysis. Low molecular weight (LMW) RNA was purified using the mirVana $^{\mathrm{TM}}$ miRNA extraction kit (Ambion, Austin, TX, USA). About $250 \mathrm{ng}$ of [23]LMW RNA was used as the input for miRNAs labelling using the Array900 miRNA Direct kit (Genisphere Inc., Hatfield, PA, USA), a tagging method that allows direct labeling of mature miRNAs without PCR amplification. All samples were labelled with Cy5 dye. A pooled control was labelled with $\mathrm{Cy} 3$ and equal amount of the tagged Cy3 was hybridized to every chip. The fluorescent (Cy5/Cy3) signal of each labelled miRNA was amplified about 850-900 times after being tagged with 3DNA dendromer in hybridization [22].

\section{MiRNA microarrays}

For microRNA expression evaluation, we used miRMAX microarray technology [22] that features tandem dimer probes complementary to mature microRNA sequences (or abridged sequence) of all the identified miRNAs of 
Drosophila according to the miRNA registry VERSION 5.1 [23]. The total number of miRNAs on the chip is 1231, which includes 1087 miRNAs from miRbase 5.1 and 144 predicted human miRNAs. The chip contains several subarrays for all miRNAs from Drosophila, C. elegans, human, mouse, and rat so it can be used for probing various organisms. We only extracted data from the Drosophila subarray. The miRMAX microarray can detect miRNA expression with only $100 \mathrm{ng}$ LMW RNA input. Three independent RNA samples for each time point were hybridized and analyzed. Microarray chips were hybridized in Hybex Microarray Incubation System (SciGene, Sunnyvale, $\mathrm{CA}$ ) and processed according to the protocol of the Array900 miRNA Direct kit (Genisphere Inc., Hatfield, PA, USA). A GenePix 4000B scanner (Axon Instruments, Union City, CA, USA) was used to scan individual chips and median spot intensities were generated using GenePix 4.0 (Axon Instruments). The Gene Expression Omnibus (GEO) accession number for our microarray raw data is GSE10005.

\section{Statistical analysis for microarrays}

Scanned microarray raw data were processed and normalized using a GeneTraffic Duo server on campus (Strategene, La Jolla, CA, USA). We used the Cy3 labelled $c y c^{01}$ ZT1 samples (About 200 ng LMW RNA input for each chip) for normalization, so that we could compare ratios of a given miRNA relative to the same sample. Data were transformed into $\log 2$ ratio and were further analyzed by using the Genespring GX software (Agilent, Santa Clara, CA). First, miRNAs from all genes were tested with Welch ANOVA among the following groups based on time points ZT1, 7, 13, and 19 in both $y w$ and $c y c^{01}$ origin; pvalue cutoff 0.05 , multiple testing correction: Benjamini and Hochberg False Discovery Rate (FDR). Then, Welch ttest was used to identify significantly differentially expressed miRNAs between "origin $y w$ " and "origin $c y c^{01}$ " from the Volcano Plot built. Multiple Testing Correction: Benjamini and Hochberg False Discovery Rate. Which miRNAs were differentially expressed was defined by Fold Difference, 1.5 and a P-value Cutoff, 0.05.

\section{Quantitative RT-PCR}

Approximately 30 to 40 adult flies were frozen on dry ice at the indicated times during either LD or DD and kept at $-80^{\circ} \mathrm{C}$ until further processing. Fly heads were isolated and crushed in $200 \mu \mathrm{l}$ of TRI Reagent (Sigma) with a motorized pestle, as previously described $[54,55]$. Total RNA was prepared according to the manufacturer's instruction and the final RNA pellet was resuspended in 12-15 $\mu \mathrm{l}$ of DEPC-treated water. For the analysis of miR263a and miR-263b, $4 \mu \mathrm{l}$ of total RNA solution were subjected to reverse transcription in the presence of miRNAspecific primers as supplied by the manufacturer (TaqMan MicroRNA Assay, Applied Biosystems). The resulting
cDNAs were diluted 15 -fold in $1 \mathrm{mM}$ Tris- $\mathrm{HCl}(\mathrm{pH} 8.0)$. Subsequently, $2 \mu \mathrm{l}$ of cDNA solution were added to a total of $20 \mu \mathrm{l}$ and PCR reactions performed in triplicate using either the miR-263a or miR-263b specific primers, according to the manufacturer's instructions (TaqMan MicroRNA Assay, Applied Biosystems). We used oligo-dT primed cDNA synthesis to measure the levels of the internal controls, $r p 49$ or $c b p 20$, as previously described [54,55]. Briefly, $2 \mu \mathrm{l}$ of total RNA solution was used for reverse transcription (Omniscript RT, Qiagen). The resulting cDNAs were diluted 10-fold in $1 \mathrm{mM}$ Tris- $\mathrm{HCl}(\mathrm{pH}$ 8.0). Subsequently, $2 \mu \mathrm{l}$ of cDNA solution were added to a total of $30 \mu \mathrm{l}$ and PCR reactions performed in triplicate (QuantiTect SYBR Green PCR, Qiagen) with the following conditions: an initial step of $15 \mathrm{~min}$ at $95^{\circ} \mathrm{C}$ to activate HotStarTaq DNA polymerase, followed by 40 cycles of 15 $\mathrm{s}$ at $94^{\circ} \mathrm{C}, 30 \mathrm{~s}$ at $60^{\circ} \mathrm{C}$, and $30 \mathrm{~s}$ at $72^{\circ} \mathrm{C}$. All PCR reactions were performed in 96-well plates using an $\mathrm{ABI}$ prism 7600 system (Applied Biosystems). Standard curves were generated with serially diluted cDNA samples for every run and the relative copy number of the gene of interest was calculated based on Ct (threshold cycle) values. The values for miR-263a and miR-263b were normalized to the relative copy number of $r p 49$ or $c b p 20$ cDNAs. Finally, the relative levels of miR-263a and miR-263b at ZT/CT1 were set to 1.0 and the other values normalized. The efficiency of all the PCR reactions was at least $90 \%$.

\section{Authors' contributions}

MY prepared the RNA samples for microarray analysis, did the microarrays and performed the statistical analysis. JL collected the flies, helped in the RNA preparation and also did the quantitative RT-PCR experiments. IE and RWP conceived of the study, supervised the work and contributed to writing the manuscript. All authors read and approved the final manuscript.

\section{Acknowledgements}

This work was partially supported by a Busch Biomedical Research Grant from Rutgers University awarded to IE and RWP, and a grant from the National Institutes of Health (NS034958) to IE.

\section{References}

I. Edery I: Circadian rhythms in a nutshell. Physiol Genomics 2000, 3(2):59-74.

2. Dunlap JC: Molecular bases for circadian clocks. Cell 1999 96(2):27I-290.

3. Gallego M, Virshup DM: Post-translational modifications regulate the ticking of the circadian clock. Nat Rev Mol Cell Biol 2007, 8(2): $139-148$

4. Duffield GE: DNA microarray analyses of circadian timing: the genomic basis of biological time. J Neuroendocrinol 2003, I5(1 0):991-1002.

5. Etter PD, Ramaswami M: The ups and downs of daily life: profiling circadian gene expression in Drosophila. Bioessays 2002, 24(6):494-498.

6. Vitalini MW, de Paula RM, Park WD, Bell-Pedersen D: The rhythms of life: circadian output pathways in Neurospora. J Biol Rhythms 2006, 2 I(6):432-444.

7. $\mathrm{Fu}$ L, Lee CC: The circadian clock: pacemaker and tumour suppressor. Nat Rev Cancer 2003, 3(5):350-36I. 
8. McClung CA: Circadian genes, rhythms and the biology of mood disorders. Pharmacol Ther 2007, I I4(2):222-232.

9. Staels B: When the Clock stops ticking, metabolic syndrome explodes. Nat Med 2006, I 2(I):54-5; discussion 55.

10. Hall JC: Genetics and molecular biology of rhythms in Drosophila and other insects. Adv Genet 2003, 48: I-280.

II. Bushati N, Cohen SM: microRNA Functions. Annu Rev Cell Dev Biol 2007.

12. Bagga S, Bracht J, Hunter S, Massirer K, Holtz J, Eachus R, Pasquinelli AE: Regulation by let-7 and lin-4 miRNAs results in target mRNA degradation. Cell 2005, 122(4):553-563.

13. Jackson RJ, Standart N: How do microRNAs regulate gene expression? Sci STKE 2007, 2007(367):rel.

14. Valencia-Sanchez MA, Liu J, Hannon GJ, Parker R: Control of translation and mRNA degradation by miRNAs and siRNAs. Genes Dev 2006, 20(5):515-524.

15. Lewis BP, Burge CB, Bartel DP: Conserved seed pairing, often flanked by adenosines, indicates that thousands of human genes are microRNA targets. Cell 2005, I 20(I): 15-20.

16. Ambros V: The functions of animal microRNAs. Nature 2004, 43 I(7006):350-355

17. Gesellchen $\mathrm{V}$, Boutros $\mathrm{M}$ : Managing the genome: microRNAs in Drosophila. Differentiation 2004, 72(2-3):74-80.

18. Jaubert S, Mereau A, Antoniewski C, Tagu D: MicroRNAs in Drosophila: The magic wand to enter the Chamber of Secrets? Biochimie 2007.

19. Rutila JE, Suri V, Le M, So WV, Rosbash M, Hall JC: CYCLE is a second bHLH-PAS clock protein essential for circadian rhythmicity and transcription of Drosophila period and timeless. Cell 1998, 93(5):805-814.

20. Helfrich-Forster C: Neurobiology of the fruit fly's circadian clock. Genes Brain Behav 2005, 4(2):65-76.

21. Hardin PE, Hall JC, Rosbash M: Feedback of the Drosophila period gene product on circadian cycling of its messenger RNA levels. Nature 1990, 343(6258):536-540.

22. Goff LA, Yang M, Bowers J, Getts RC, Padgett RW, Hart RP. Rational probe optimization and enhanced detection strategy for microRNAs using microarrays. RNA Biol 2005, 2(3):93-100.

23. miRBase [http://microrna.sanger.ac.uk]

24. Glossop NR, Lyons LC, Hardin PE: Interlocked feedback loops within the Drosophila circadian oscillator. Science 1999, 286(5440):766-768.

25. Grimson A, Farh KK, Johnston WK, Garrett-Engele P, Lim LP, Bartel DP: MicroRNA targeting specificity in mammals: determinants beyond seed pairing. Mol Cell 2007, 27(I):9I-105.

26. PicTar [http://pictar.bio.nyu.edu]

27. TargetScan: Prediction of microRNA targets [http:// www.targetscan.org]

28. miRNA - Target Gene Prediction at EMBL [http://www.rus sell.embl.de/miRNAs]

29. Ceriani MF, Hogenesch JB, Yanovsky M, Panda S, Straume M, Kay SA: Genome-wide expression analysis in Drosophila reveals genes controlling circadian behavior. J Neurosci 2002 22(21):9305-9319.

30. Claridge-Chang A, Wijnen H, Naef F, Boothroyd C, Rajewsky N, Young MW: Circadian regulation of gene expression systems in the Drosophila head. Neuron 200I, 32(4):657-67I.

31. Keegan KP, Pradhan S, Wang JP, Allada R: Meta-Analysis of Drosophila Circadian Microarray Studies Identifies a Novel Set of Rhythmically Expressed Genes. PLoS Comput Biol 2007, 3(II):e208.

32. Lin Y, Han M, Shimada B, Wang L, Gibler TM, Amarakone A, Awad TA, Stormo GD, Van Gelder RN, Taghert PH: Influence of the period-dependent circadian clock on diurnal, circadian, and aperiodic gene expression in Drosophila melanogaster. Proc Natl Acad Sci U S A 2002, 99(14):9562-9567.

33. McDonald MJ, Rosbash M: Microarray analysis and organization of circadian gene expression in Drosophila. Cell 2001, 107(5):567-578.

34. Ueda HR, Matsumoto A, Kawamura M, lino M, Tanimura T, Hashimoto S: Genome-wide transcriptional orchestration of circadian rhythms in Drosophila. I Biol Chem 2002, 277( ( 6): | $4048-14052$.
35. Wijnen $\mathrm{H}$, Naef F, Young MW: Molecular and statistical tools for circadian transcript profiling. Methods Enzymol 2005, 393:34I-365.

36. Bae K, Lee C, Sidote D, Chuang KY, Edery I: Circadian regulation of a Drosophila homolog of the mammalian Clock gene: PER and TIM function as positive regulators. Mol Cell Biol 1998 , I8(I0):6|42-6I5|.

37. Kim EY, Edery I: Balance between DBT/CKIepsilon kinase and protein phosphatase activities regulate phosphorylation and stability of Drosophila CLOCK protein. Proc Natl Acad Sci U S A 2006, 103(16):6178-6183.

38. Yu W, Zheng H, Houl JH, Dauwalder B, Hardin PE: PER-dependent rhythms in CLK phosphorylation and E-box binding regulate circadian transcription. Genes Dev 2006, 20(6):723-733.

39. Kadener $S$, Stoleru $D$, McDonald M, Nawathean $P$, Rosbash $M$ : Clockwork Orange is a transcriptional repressor and a new Drosophila circadian pacemaker component. Genes Dev 2007, 2I(I3): I675-I686.

40. Lim C, Chung BY, Pitman JL, McGill JJ, Pradhan S, Lee J, Keegan KP, Choe J, Allada R: Clockwork orange encodes a transcriptional repressor important for circadian-clock amplitude in Drosophila. Curr Biol 2007, I7( ( 2): 1082-1089.

4I. Matsumoto A Ukai-Tadenuma M, Yamada RG, Houl J Uno KD Kasukawa T, Dauwalder B, Itoh TQ, Takahashi K, Ueda R, Hardin PE, Tanimura T, Ueda HR: A functional genomics strategy reveals clockwork orange as a transcriptional regulator in the Drosophila circadian clock. Genes Dev 2007, 2 I(I3): | 687- I700.

42. Bae K, Edery I: Regulating a circadian clock's period, phase and amplitude by phosphorylation: insights from Drosophila. J Biochem (Tokyo) 2006, I 40(5):609-617.

43. Sathyanarayanan S, Zheng X, Xiao R, Sehgal A: Posttranslational regulation of Drosophila PERIOD protein by protein phosphatase 2A. Cell 2004, I I6(4):603-6I5.

44. Kloss B, Rothenfluh A, Young MW, Saez L: Phosphorylation of period is influenced by cycling physical associations of double-time, period, and timeless in the Drosophila clock. Neuron 200I, 30(3):699-706.

45. Akten B, Jauch E, Genova GK, Kim EY, Edery I, Raabe T, Jackson FR: A role for CK2 in the Drosophila circadian oscillator. Nat Neurosci 2003.

46. Lin JM, Kilman VL, Keegan K, Paddock B, Emery-Le M, Rosbash M, Allada R: A role for casein kinase 2alpha in the Drosophila circadian clock. Nature 2002, 420(69 I 7):8|6-820.

47. Hobert O: Common logic of transcription factor and microRNA action. Trends Biochem Sci 2004, 29(9):462-468.

48. Hardin PE: The circadian timekeeping system of Drosophila. Curr Biol 2005, I 5(I7):R7|4-22.

49. Venturini L, Battmer K, Castoldi M, Schultheis B, Hochhaus A, Muckenthaler MU, Ganser A, Eder M, Scherr M: Expression of the miR17-92 polycistron in chronic myeloid leukemia (CML) CD34+ cells. Blood 2007, I 09( I 0):4399-4405.

50. Xu S, Witmer PD, Lumayag S, Kovacs B, Valle D: MicroRNA (miRNA) transcriptome of mouse retina and identification of a sensory organ-specific miRNA cluster. J Biol Chem 2007, 282(34):25053-25066.

51. Stark A, Lin MF, Kheradpour P, Pedersen JS, Parts L, Carlson JW, Crosby MA, Rasmussen MD, Roy S, Deoras AN, Ruby JG, Brennecke J, Hodges E, Hinrichs AS, Caspi A, Paten B, Park SW, Han MV, Maeder ML, Polansky BJ, Robson BE, Aerts S, van Helden J, Hassan B, Gilbert DG, Eastman DA, Rice M, Weir M, Hahn MW, Park Y, Dewey CN, Pachter L, Kent W], Haussler D, Lai EC, Bartel DP, Hannon G], Kaufman TC, Eisen MB, Clark AG, Smith D, Celniker SE, Gelbart WM, Kellis M, Crosby MA, Matthews BB, Schroeder AJ, Gramates LS, St Pierre SE, Roark M, Wiley KLJr., Kulathinal RJ, Zhang P, Myrick KV, Antone JV, Gelbart WM, Carlson JW, Yu C, Park S, Wan KH, Celniker SE: Discovery of functional elements in 12 Drosophila genomes using evolutionary signatures. Nature 2007, 450(7167):219-232.

52. Cheng HY, Papp JW, Varlamova O, Dziema H, Russell B, Curfman JP, Nakazawa T, Shimizu K, Okamura H, Impey S, Obrietan K: microRNA modulation of circadian-clock period and entrainment. Neuron 2007, 54(5):8I3-829.

53. Zeng $\mathrm{H}$, Hardin PE, Rosbash M: Constitutive overexpression of the Drosophila period protein inhibits period mRNA cycling. Embo J 1994, I3(15):3590-3598. 
54. Chen WF, Majercak J, Edery I: Clock-gated photic stimulation of timeless expression at cold temperatures and seasonal adaptation in Drosophila. J Biol Rhythms 2006, 2 I (4):256-27I.

55. Majercak J, Chen WF, Edery I: Splicing of the period gene 3'-terminal intron is regulated by light, circadian clock factors, and phospholipase C. Mol Cell Biol 2004, 24(8):3359-3372.

Publish with Biomed Central and every scientist can read your work free of charge

"BioMed Central will be the most significant development for disseminating the results of biomedical research in our lifetime. " Sir Paul Nurse, Cancer Research UK

Your research papers will be:

- available free of charge to the entire biomedical community

- peer reviewed and published immediately upon acceptance

- cited in PubMed and archived on PubMed Central

- yours - you keep the copyright

Submit your manuscript here:

http://www.biomedcentral.com/info/publishing_adv.asp
BioMedcentral 